\title{
Web-based administration of a personality questionnaire: Comparison with traditional methods
}

\author{
ROBERT N. DAVIS \\ University of Michigan, Ann Arbor, Michigan
}

\begin{abstract}
The World-Wide Web holds great promise as a mechanism for questionnaire-based research. But are data from Web-based questionnaires comparable to data from standard paper-and-pencil questionnaires? This study assessed the equivalence of the Ruminative Responses Scale in a Web-based format and in a paper-and-pencil format among introductory psychology, upper-level psychology, and nonpsychology students. Intermal consistency coefficients were comparable across the groups. The participants in the Web sample reported higher levels of self-focused rumination than did the other groups. Women in the Web sample reported more self-focused rumination than did women in the other groups. In the Web sample, results did not covary with access location. These results suggest that findings from Web-based questionnaire research are comparable with results obtained using standard procedures. The computerized Web interface may also facilitate self-disclosure among research participants.
\end{abstract}

The use of computers in psychological assessment has increased steadily over the last 30 years (Burke, 1992). The advent of the World-Wide Web offers unprecedented opportunities for researchers to benefit from the use of computers in data collection (Schmidt, 1997; Smith \& Leigh, 1997). As a research medium, the Web offers researchers at least four major benefits. First, the Web permits individuals to send data to a researcher at their convenience, in terms of time and location, which increases the potential number of eligible research participants (Schmidt, 1997; Smith \& Leigh, 1997). Second, the Web permits automatic transformation of raw data into an analyzable format, such as an SPSS data file, using procedures such as CGI scripts (see Kieley, 1996; Schmidt, Hoffman, \& MacDonald, 1997). Third, Web-based research is efficient in terms of time and the resources it requires (Schmidt, 1997). Fourth, the Web provides a degree of anonymity to research participants, which decreases the influence of demand characteristics by facilitating self-disclosure and eliminating observer bias (Esposito, Agard, \& Rosnow, 1984; Hewson, Laurent, \& Vogel, 1996; Meszaros, Engelsmann, Meterissian, \& Kusalic, 1995; Smith \& Leigh, 1997).

Is research conducted over the Web equivalent to research conducted in more traditional settings, such as a psychology laboratory? Validity studies of Web-based research have recently begun to examine this question and

This research was supported by U.S. Public Health Grant 51817 to Susan Nolen-Hoeksema. I thank Michael Bishop, Jamie Polito, and Andrew Ward for their assistance with data collection. I also thank Tom Buchanan for his helpful comments on an earlier draft of this manuscript. Correspondence concerning this article should be addressed to R. N. Davis, who is now at the University of Houston, Department of Psychology, Houston, TX 77204-5341 (e-mail: robnd@uh.edu). have reported favorable results (e.g., Krantz, Ballard, \& Scher, 1997). For example, the results from one experiment that was conducted both on the Web and in a laboratory were nearly perfectly correlated (Krantz et al., 1997). Although only a few published studies have examined the equivalence question with respect to Webbased versus paper-and-pencil administration of personality questionnaires, nascent studies in this area have reported comparable results across domains (Buchanan \& Smith, in press; Pasveer \& Ellard, 1998; Smith \& Leigh, 1997; Stanton, 1998). For example, Buchanan and Smith administered the revised version of the Self-Monitoring Scale (SMS-R; Gangestad \& Snyder, 1985) to Web users on line and also to a local student sample in paper-andpencil format. The factor structure of the SMS-R and coefficient alphas were found to be comparable across the groups, with the Web version yielding some superior results (e.g., coefficient alpha), as compared with previously reported studies (Buchanan \& Smith, 1999). Another study that compared Web-based and paper-andpencil versions of an organizational justice scale found a similar factor structure across the two domains, as well as more item variability and less missing data in the Webbased condition (Stanton, 1998).

The advent of Web-based data collection is a direct extension of data collection on stand-alone computers (Buchanan \& Smith, in press). Given the current dearth of findings pertaining to Web-based data collection, studies of computerized (non-Web-based) versus paper-andpencil data collection procedures may provide useful information. Research comparing computerized and paperand-pencil assessments has generally found that research participants prefer computerized assessments and are equally, if not more, willing to report sensitive information to a computer than to a paper-and-pencil questionnaire 
or to a human interviewer (Booth-Kewley, Edwards, \& Rosenfeld, 1992; Evan \& Miller, 1969; Finegan \& Allen, 1994; Hile \& Adkins, 1997; King \& Miles, 1995; Millstein, 1987; Petrie \& Abell, 1994; Wilson, Genco, \& Yager, 1986). In one study, recently admitted parasuicide patients preferred a computerized interview to an interview with a clinician (Petrie \& Abell, 1994). In another study, recent suicide attempters disclosed more information to computerized depression and suicidal ideation questionnaires than to a clinician interviewer (Levine, Ancill, \& Roberts, 1989). Moreover, the computerized measures were better predictors of suicidality than was the clinician interviewer (Levine et al., 1989). People also appear to be more willing to report behaviors such as substance use to a computer than to a clinician or a paper-and-pencil questionnaire (Lucas, 1977; Lucas, Mullin, Luna, \& McInroy, 1977; Skinner \& Allen, 1983). Although these findings are informative, the extent to which they generalize to Web-based research is an open question.

Despite the initial promising evidence in support of Web-based psychological research (e.g., Buchanan \& Smith, in press; Krantz et al., 1997; Michalak, 1998; Pasveer \& Ellard, 1998; Smith \& Leigh, 1997; Stanton, 1998), it is necessary to demonstrate the equivalence of Web-based and traditional data collection methods empirically before adopting Web-based methods extensively (Krantz et al., 1997; Smith \& Leigh, 1997). There remain numerous potential threats to the reliability and validity of Web-based questionnaires (see Hewson et al., 1996; Schmidt, 1997, for reviews). For example, participants recruited from the Web are likely to be more heterogeneous on demographic and other variables than participants recruited by traditional means (Buchanan \& Smith, in press). Moreover, researchers lose considerable control over the testing environment when conducting Web-based research. Some participants may send data from a quiet room, whereas others might send it from a noisy computer laboratory (Reips, 1996). In addition, Web-based research is open to exploitation by people who participate numerous times and/or provide disingenuous data (Schmidt, 1997; Smith \& Leigh, 1997). The primary concern underlying these possibilities is that factors inherent in the Web interface, the Web sample, and/or the testing environment might obfuscate the extent to which an otherwise useful measure with good psychometric properties is able to measure the construct of interest. In light of these concerns, several relevant analyses will be performed in the present study (see below).

The present study assessed the equivalence of a Webbased versus a paper-and-pencil administration of a personality questionnaire commonly used as a screening instrument: the Ruminative Responses Scale (RRS) from the Response Styles Questionnaire (RSQ; Nolen-Hoeksema \& Morrow, 1991). The screening of participants for research and their subsequent selection on the basis of inclusion and exclusion criteria is a common practice among researchers on college campuses and in medical settings.
Participants scoring at the upper and/or the lower end of an overall distribution on variables of interest are frequently selected for follow-up studies on the basis of results from an initial screening session-for example, a tendency to engage in self-focused rumination when experiencing dysphoric affect (cf. Davis \& Nolen-Hoeksema, in press). These procedures facilitate research on interactions between subject variables, such as personality traits, and experimental treatments (cf. Lyubomirsky \& Nolen-Hoeksema, 1993, 1995), as well as cross-sectional studies of variables on which an experimenter wants to select participants (e.g., age or gender).

The measure used in the present study assesses the extent to which people tend to respond to feelings of sadness or depression with self-focused rumination. Selffocused rumination describes a tendency to engage in passive thoughts and behaviors that focus one's attention on one's depressed mood and on the implications of these symptoms, rather than taking action to alleviate one's symptoms (see Nolen-Hoeksema, 1990, 1991, for reviews). People who ruminate may tend to sit alone and think about how tired and unmotivated they feel or passively review all the negative happenings in their lives, without taking action to change their situation. The RSQ (NolenHoeksema \& Morrow, 1991) was developed to assess people's habitual responses to depressed mood, and the RRS consists of a series of items on the RSQ that assess the tendency to engage in self-focused rumination. A short (10-item) form of the RRS was recently developed and was used in the present study (cf. Davis \& Nolen-Hoeksema, in press).

In the present study, the RRS was administered to four samples of participants: (1) introductory psychology students; (2) upper-level psychology students; (3) nonpsychology students; and (4) a Web-based student sample. The nonpsychology and upper-level psychology student groups were included in order to determine whether they differed from introductory psychology students even when using the same methods (i.e., paper and pencil) for filling out the questionnaire. The Web sample was deliberately drawn only from the local student population, in order to ensure comparability with the other groups on major demographic variables. This procedure enables a comparison of group differences as a function of the assessment medium (i.e., Web vs. paper and pencil), while limiting the heterogeneity that might result from a general Web sample. Moreover, within the Web sample itself, we analyzed possible differences as a function of the location from which the participants accessed the Web (e.g., at home vs. a campus computer laboratory). These analyses were performed to determine whether concerns about loss of experimental control over the testing setting were warranted-whether responses to the questionnaire differed systematically as a function of the participant's location.

It was predicted that the Web sample would report a greater tendency to engage in self-focused rumination, as compared with the other three groups. These predictions 
were based on previous findings that people are generally more willing to report sensitive information to a computer than to a paper-and-pencil questionnaire. Moreover, it was expected that decreased observer bias and increased anonymity would facilitate self-disclosure in the Web sample. Gender was also included as an independent variable, in light of previous findings that women are more likely to ruminate when depressed than are men (e.g., Schwartz \& Koenig, 1996; Thayer, Newman, \& McClain, 1994). Moreover, there are gender differences in Web usage (GVU Center, 1998). Thus, the possible interaction between sample group and gender was also examined.

\section{METHOD}

\section{Participants}

Four groups of individuals participated in the study: (1) 128 students ( 56 males, 72 females) from the Web; (2) 118 students (46 males, 72 females) from upper-level psychology courses; (3) 113 students ( 50 males, 63 females) from nonpsychology courses; and (4) a total of 1,012 students ( 427 males, 585 females) from introductory psychology courses. The participants ranged in age from 17 to 26 years and were drawn from the University of Michigan campus. In the nonpsychology sample, participants were recruited from three lecture courses: History of the United States: 1865present; Planets and Moons (Geology); and Introductory Biology. The upper-level psychology sample was also recruited from three lecture courses: Personality Psychology, Social Psychology, and Abnormal Psychology. The Web participants were recruited from flyers posted around the university campus that included the study's URL (Internet address). A Pearson chi-square test indicated that the groups did not differ significantly in sex composition $\left[\chi^{2}(3)=.836\right.$, $p>.10, N=1,371]$.

\section{Materials}

Demographics questionnaire. The participants filled out a one-page demographics questionnaire and provided information about their age, sex, native language, and contact information.

Ruminative Responses Scale. A shortened (10-item) form of the RRS of the RSQ was used in this study. This scale assesses how participants tend to respond to their own symptoms of negative emotion. The instructions for this questionnaire read:

People think and do many different things when they feel sad, blue, or depressed. Please read each of the items below and indicate whether you never, sometimes, often, or always think or do each one when you feel sad, down, or depressed. Please indicate what you generally do, not what you think you should do.

The original RRS includes 22 items describing responses to depressed mood that are self-focused (e.g., I think "Why do I react this way?"), symptom-focused (e.g., I think about how hard it is to concentrate), and focused on the possible causes and consequences of one's mood (e.g., I think "I won't be able to do my job if I don't snap out of this"). Scores on this scale show high test-retest reliability $(r=.80$ at 6 months, $r=.66$ at 1 year; Nolen-Hoeksema \& Larson, 1998; Nolen-Hoeksema, Parker, \& Larson, 1994), internal consistency (Cronbach's alpha $=.89$; Nolen-Hoeksema \& Morrow, 1991), and acceptable convergent and predictive validity (Butler \& Nolen-Hoeksema, 1994; Nolen-Hoeksema \& Morrow, 1991; NolenHoeksema et al., 1994). The 10 items on this shortened scale were based on item analyses conducted with a community sample of 1,122 adults (Nolen-Hoeksema, unpublished data). The 10 items from the RRS that correlated most strongly with total scores on the longer scale, and on which at least $15 \%$ of the sample endorsed an answer other than never, were chosen for inclusion on the shortened scale. The internal consistency (Cronbach's alpha) of this 10-item scale in the community sample of 1,122 was .87 , and the correlation between this 10-item scale and the total 22-item scale was .93.

\section{Procedure}

Paper administration. The investigators received permission from instructors in large lecture courses to visit their respective classes (listed above) in order to solicit student participation in a research study. Both the course lecturers and the participants were blind as to the purpose of the study. In all cases, all but one to three students in each lecture course participated in the study. All the participants provided informed consent and received a debriefing after completing the questionnaire. The participants in the introductory psychology class received course credit for completing the forms, and those in the other classes were paid $\$ 5$ for completing the forms. In addition, the participants were instructed to print and sign their names on a research recruitment sheet at the end of the questionnaire packet if they were interested in possibly being selected to participate in other studies for pay.

Web administration. The participants responded to flyers on campus advertising a research study that required visiting a Web site and filling out a questionnaire on line. Interested participants tore off a piece of the flyer that included the study's URL and then used a Web browser to visit the Web site constructed for questionnaire administration. The Web site included a copy of the consent form, as well as the demographics questionnaire and RRS. The Web page also informed participants that they would have a chance of being selected to participate in another study for pay by filling out the questionnaires. Thus, the participants in each of the four groups had some incentive for completing the forms. The participants typed the information requested by the demographic questionnaire onto the Web page and used a mouse to click on their responses to the RRS. Only one response per questionnaire item was permitted, and the participants had the ability to scroll up or down on the Web page. After filling out the questionnaires, the participants used a mouse to click on one of two text boxes, "Send Form" or "Clear Form." Clicking on "Send Form" sent the data to the experimenter by means of e-mail. When this occurred, the participants then saw a screen indicating that their message had been sent successfully. Clicking on "Clear Form" erased the data completely. This option was provided in the event that participants opted not to submit their data or wished to clear the page and start over. The Web page forms were converted to e-mail message format, using the program $\mathrm{Ht}$ mail (Disser, 1996).

In light of concerns that the participants from the lecture classes could also have participated in the Web portion of the study, and given that it was possible for some people to complete the questionnaire more than once on line, research assistants checked the data before the analyses to ensure that no data were entered for the same participant twice. These data-cleaning procedures resulted in the deletion of data from 2 participants who completed the questionnaire on line more than once (entries following the first instance of participation were viewed as spurious). Data were also deleted from 1 participant who completed the questionnaires both on line and in two of her classes. These procedures resulted in four independent samples.

\section{RESULTS}

\section{Internal Consistency}

To examine the psychometric properties of the Webbased RRS, as compared with the paper-and-pencil version, the internal consistency (Cronbach's alpha) of the RRS was assessed separately for the four samples. Cronbach's alphas for the RRS were as follows: .82 (Web sam- 
ple), .88 (upper-level psychology students), .88 (nonpsychology students), and .83 (introductory psychology students).

\section{Group and Gender Differences}

Sample group and gender differences were assessed, using a 2 (gender) $\times 4$ (group) analysis of variance (ANOVA), with sample group (Web sample, upper-level psychology students, nonpsychology students, and introductory psychology students) and gender as independent variables and RRS total scores as dependent variables. Table 1 presents the means and standard deviations for all scores by sample group and gender. There were significant group differences on RRS scores $[F(3,1363)=$ $4.23, p<.011$. Post hoc follow-up tests, using Tukey's honestly significant differences (HSD) tests, revealed that students in the Web sample reported levels of rumination that were significantly higher than those reported by students in both introductory psychology $(p<.01)$ and nonpsychology $(p<.01)$ courses, but not those reported by students in upper-level psychology courses $(p>.10)$. Significant gender differences were also obtained on RRS scores $[F(1,1363)=8.47, p<.01]$, indicating that women reported significantly greater rumination than did men.

In addition, there was a nonsignificant trend toward a group $\times$ gender interaction on $\mathrm{RRS}$ scores $[F(3,1363)=$ $2.22, p<.09]$. Follow-up one-way ANOVAs within each gender separately revealed no significant group differences among males $[F(3,575)=0.53, p>.10]$, but did reveal significant group differences among females $[F(3,788)=6.70, p<.0001]$. Post hoc follow-up tests, using Tukey's HSD, revealed that women in the Web sample reported significantly greater rumination than did women in introductory psychology $(p<.0001)$ and nonpsychology $(p<.0001)$ courses. Women in the Web sample also reported greater rumination than did women in upper-level psychology courses, but the difference was not statistically significant $(p<.08)$.

\section{Analysis of Web Data by Access Location}

The 128 participants who filled out the RRS on the Web opted to do so from a variety of locations across campus. The server program used in this study (Htmail) also automatically detected and reported the terminal location from which the data originated. In the Web sample, $13 \%$ $(n=17)$ used a modem to access the Web page from home, $49 \%(n=63)$ accessed the Web page from a computing site on campus, and $38 \%(n=48)$ accessed the

Table 1

Means and Standard Deviations of Scores on the Ruminative Responses Scale

\begin{tabular}{|c|c|c|c|c|c|c|}
\hline \multirow[b]{2}{*}{ Group } & \multicolumn{2}{|c|}{ Total } & \multicolumn{2}{|c|}{ Men } & \multicolumn{2}{|c|}{ Women } \\
\hline & $M$ & $S D$ & $M$ & $S D$ & $M$ & $S D$ \\
\hline Web & 11.31 & 4.76 & 9.64 & 4.47 & 12.61 & 4.60 \\
\hline Psychology & 10.41 & 5.03 & 10.02 & 4.30 & 10.65 & 5.45 \\
\hline Introductory psychology & 9.83 & 4.91 & 9.45 & 4.97 & 10.11 & 4.85 \\
\hline Nonpsychology & 9.04 & 4.97 & 8.80 & 4.52 & 9.24 & 5.32 \\
\hline
\end{tabular}

Web page from a computer on campus that was not part of a public computing site (e.g., from a science laboratory or an office). Follow-up analyses using a one-way ANOVA, with access location (home, campus computing site, or other campus computer) as the independent variable and RRS total scores as dependent variables, found no significant differences across access locations $[F(2,125)=1.34, p>.10]$.

\section{DISCUSSION}

In this study, students who completed a personality questionnaire on the Web reported higher levels of selffocused rumination than did students who completed the same questionnaire in paper-and-pencil format. The internal consistency of the Web version was comparable with the paper-and-pencil versions. Although students in the Web sample participated in the study from varying locations, this difference did not affect their responses in any systematic manner. Taken together, these findings support the use of the RRS on the Web and provide tentative evidence that the Web is a viable mechanism for questionnaire-based data collection and/or subject screening.

The participants in the Web sample reported more selffocused rumination than did the participants who filled out paper-and-pencil questionnaires, although the Web sample did not differ from the students in upper-level psychology courses. Although women reported higher levels of rumination than did men in the general sample (collapsed across group), women in the Web sample also reported more rumination than did women who filled out paper-and-pencil questionnaires. This finding might reflect increased self-disclosure among women in the context of a Web-based questionnaire. Some women may feel more open to disclose tendencies such as ruminative coping in a context that is arguably less evaluative than a typical data collection context. By allowing people to have more control over their environment when they disclose information about sensitive issues, such as how one responds to depressed mood, Web-based questionnaires may encourage increased frankness of response and selfdisclosure (Hewson et al., 1996). A further possibility is that some people may gain greater access to their feelings and personality when completing a Web-based questionnaire, as compared with a questionnaire in a large lecture class. Given that women are more likely than men to ruminate when depressed, this tendency may be facilitated in the context of a Web-based questionnaire.

The internal consistency of the RRS was similar across the four groups. This finding indicates that these questionnaires consistently measure a single construct and that results from the Web version are comparable with those obtained in the other samples. The Web versions of the RRS also were unaffected by differences in the location from which the participants accessed the Web. This finding suggests that the data people provide to a Web page do not differ significantly as a function of whether 
they choose to fill out questionnaires at home, a public computing site, or another computer on campus. Although permitting access from multiple locations decreases experimental control, it does not appear to affect questionnaire results adversely.

A highly publicized study recently suggested that Internet usage was associated with increases in depression and loneliness and with declines in family communication and the size of participants' social circles (Kraut et al., 1998). This finding raises the question of whether the present results reflect a sampling artifact, rather than differences that are a function of the assessment medium. Although such a possibility cannot be ruled out definitively, it is unlikely to have occurred for several reasons. First, the present study sampled from college students with a working knowledge of Web browsing, rather than from first-time Internet users. Second, the findings of Kraut et al. demonstrate only small relationships at best (e.g., the largest correlation between Internet usage and adjustment measures is .19). Third, although Kraut et al.'s data were interpreted as showing that participants used the Internet as their social and emotional functioning declined, Internet usage may have provided users with a newfound hobby or a constructive activity to engage in given that social and emotional functioning had declined, rather than having caused the social and emotional difficulties. Thus, there remains no conclusive or causal evidence that Internet users have more social and/or emotional problems than non-Internet users, nor has it been established definitively that Internet use causes social and/or emotional problems. The lack of such evidence and the dissimilarity of the samples used in this study and in that of Kraut et al. suggest that the present results are not simply a sampling artifact.

It might be argued that the Web sample reported spuriously higher levels of self-focused rumination than did the other groups owing to computer anxiety (cf. Tseng, Tiplady, MacLeod, \& Wright, 1998). This suggestion is unlikely for three reasons. First, the participants in the Web sample volunteered to participate in this study, which required filling out a questionnaire on line. It is not likely that computer-anxious people would volunteer for such a study. Second, previous research has found that greater levels of education are associated with lower computer anxiety (Igbaria \& Parasuraman, 1989). All the participants in the present study were college students attending a highly competitive university. Third, the RRS is not a symptom questionnaire and has no a priori relationship to computer anxiety. Rather, it measures the relatively stable tendency to respond to depressed mood with passive, emotion-focused thoughts and behaviors.

Alternatively, it could be argued that differences among the groups other than the assessment medium might account partly or fully for the observed results. Specifically, the Web sample replied to a general advertisement, whereas the other groups were solicited at a lecture. Thus, the Web sample may have been more motivated and more interested in participating. It should be noted, however, that flyers advertising the study were strategically placed near computer laboratories and other computer facilities to minimize inconvenience to potential participants. Many people were observed simply writing down the URL as they went to check their e-mail or to perform other routine computing tasks. Thus, both the Web sample and the paper-and-pencil samples were solicited at comparable, although not identical, moments of convenience.

This study provides some initial data that tend to support the use of the Web for questionnaire-based research. However, more validation of Web-based research is needed (Krantz et al., 1997). The results of the present study increase confidence in the use of the Web as a screening device and as a method for collecting questionnaire data. However, the participants were sampled from a college campus and were restricted in terms of age range. More data, using multiple measures and a broader sample, would be desirable in future studies. In addition, experimental studies of the mechanisms underlying participants' higher scores in the Web group would be useful. Identification of such mechanisms and the continued validation of the Web as a research tool appear to be growing areas of research (Schmidt, 1997; Smith \& Leigh, 1997).

\section{REFERENCES}

Booth-Kewley, S., Edwards, J. E., \& Rosenfeld, P. (1992). Impression management, social desirability, and computer administration of attitude questionnaires: Does the computer make a difference?. Journal of Applied Psychologv, 77, 562-566.

Buchanan, T., \& Smith, J. L. (1999). Using the Internet for psychological research: Personality testing on the World-Wide Web. British Journal of Psychology, 90, 125-144.

Burke, M. J. (1992). Computerized psychological testing: Impacts on measuring predictor constructs and future job behavior. In N. Schmitt \& W. C. Borman (Eds.), Personnel selection in organizations (pp. 203238). San Francisco: Jossey-Bass.

Butler, L. D., \& Nolen-Hoeksema, S. (1994). Gender differences in responses to a depressed mood in a college sample. Sex Roles, 30 , $331-346$.

Davis, R. N., \& Nolen-Hoeksema, S. (in press). Cognitive inflexibility among ruminators and nonruminators. Cognitive Therapy \& Research.

DisSER, D. (1996). Htmail: A generic forms-to-email gateway [Computer program]. Ann Arbor: University of Michigan Information Technology Division.

Esposito, J., Agard, E., \& Rosnow, R. (1984). Can confidentiality of data pay off? Personality \& Individual Differences, 5, 744-745.

Evan, W. M., \& Miller, J. R. (1969). Differential effects on response bias of computer vs. conventional administration of a social science questionnaire: An exploratory methodological experiment. Behavioral Science, 14, 216-227.

Finegan, J. E., \& Allen, N. J. (1994). Computerized and written questionnaires: Are they equivalent? Computers in Human Behavior, 10, 483-496.

Gangestad, S. W., \& SNyder, M. (1985). "To carve nature at its joints": On the existence of discrete classes in personality. Psychological Review, 92, 317-340.

Graphics, Visualization, and Usability Center (1998). GVU's $9 t h$ WWW survey results [On-line]. Atlanta: Georgia Institute of Technology, College of Computing. Available URL: http://www.cc.gatech.edu/gvu/user_surveys/

Hewson, C. M., Laurent, D., \& Vogel, C. M. (1996). Proper method- 
ologies for psychological and sociological studies conducted via the Internet. Behavior Research Methods, Instruments, \& Computers, 28 186-191.

HiLe, M. G., \& AdKins, R. E. (1997). Do substance abuse and mental health clients prefer automated assessments? Behavior Research Methods, Instruments, \& Computers, 29, 146-150.

IgBaria, M.. \& Parasuraman, S. (1989). A path analytic study of individual characteristics, computer anxiety, and attitudes toward microcomputers. Journal of Management, 15, 373-388.

KIELEY, J. M. (1996). CGI scripts: Gateways to World-Wide Web power Behavior Research Methods. Instruments, \& Computers, 28, 165-169.

KING, W. C., \& MiLEs, E. W. (1995). A quasi-experimental assessment of the effect of computerizing noncognitive paper-and-pencil measurements: A test of measurement equivalence. Journal of Applied Psychology, 80, 643-65I.

Krantz, J. H., Ballard, J., \& SCHER, J. (1997). Comparing the results of laboratory and World-Wide Web samples on the determinants of female attractiveness. Behavior Research Methods, Instruments, \& Computers, 29, 264-269.

Kraut, R., Patterson, M., Lundmark, V., Kiesler, S., MukophaDHYAY, T., \& SCHERLIS, W. (1998). Internet paradox: A social technology that reduces social involvement and psychological well-being? American Psychologist, 53, 1017-1031.

LEVINE, S., ANCILL, R. J., \& RoBERTS, A. P. (1989). Assessment of suicide risk by computer-delivered self-rating questionnaire: Preliminary findings. Acta Psychiatrica Scandinavica, 80, 216-220.

LUCAS. R. W. (1977). A study of patients' attitudes to computer interrogation. International Journal of Man-Machine Studies, 9. 69-86.

LuCas, R. W., Mul.lin, P. J., Luna, C. B. X., \& MCINROY. D. C. (1977). Psychiatrists and a computer as interrogators of patients with alcoholrelated illnesses: A comparison. British Journal of Psychiatry, 131, 160-167.

LyUbomirsky. S. \& Nolin-Hofksema. S. (1993). Self-perpetuating properties of dysphoric rumination. Journal of Personality \& Social Psychology, 65, 339-349.

LyubomiRSKy, S.. \& NOLEN-Hoeksema, S. (1995). Effects of selffocused rumination on negative thinking and interpersonal problem solving. Journal of Persomality \& Social Pswchology, 69. 176-190.

Meszaros, A.. Engel.smann. F.. Meterissian, G., \& Kusalic, M. (1995). Computerized assessment of depression and suicidal ideation. Journal of Nervous \& Mental Disease, 183, 487-488.

Michalak, E. E. (1998). The use of the Internet as a research tool: The nature and characteristics of seasonal affective disorder (SAD) amongst a population of users. Interacting with Computers, 9, 349-365.

Mit.L.STEIN, S. G. (1987). Acceptability and reliability of sensitive information collected via computer interview. Educational \& Psvchological Measurement, 47, 523-533.

Nolen-Hofksema. S. (1990). Sex differences in depression. Palo Alto, CA: Stanford University Press.

NOL.EN-HoEkSEMA. S. (1991). Responses to depression and their effects on the duration of depressive episodes. Journal of Abnormal Psychologv, 100, 569-582.

Nolen-Hofksema, S., \& LARSON, J. (1998). A dynamic model of the gender differences in depressive symptoms. Manuscript submitted for publication.

Nolen-Hoeksema, S.. \& Morrow, J. (1991). A prospective study of depression and posttraumatic stress symptoms after a natural disaster: The 1989 Loma Prieta earthquake. Journal of Personality \& Social Psychology, 61, 115-121.

Nolen-Hoeksema, S., Parker, L., \& Larson, J. (1994). Ruminative coping with depressed mood following loss. Journal of Personality \& Social Psychology. 67, 92-104.

Pasveer, K. A., \& Ellard, J. H. (1998). The making of a personality inventory: Help from the WWW. Behavior Research Methods, Instruments, \& Computers, 30, 309-313.

PETRIE, K., \& ABELL, W. (1994). Responses of parasuicides to a computerized interview. Computers in Human Behavior, 10, 415-418.

REIPS, U. (1996, October). Experimenting in the World Wide Web. Paper presented at the Society for Computers in Psychology conference, Chicago.

SchmidT, W. C. (1997). World-Wide Web survey research: Benefits, potential problems, and solutions. Behavior Research Methods, Instruments, \& Computers, 29, 274-279.

Schmidt, W. C., Hoffman, R., \& MacDonald, J. (1997). Operate your own World-Wide Web server. Behavior Research Methods, Instruments, \& Computers, 29, 189-193.

Schwartz, J. A. J., \& KoEnIG, L. J. (1996). Response styles and negative affect among adolescents. Cognitive Therapy \& Research, 20, 13-36.

Skinner, H. A., \& Allen, B. A. (1983). Does the computer make a difference? Computerized versus self-report assessment of alcohol, drug, and tobacco use. Journal of Consulting \& Clinical Psychology, 51, 267-275.

SMITH, M. A.. \& LEIGH, B. (1997). Virtual subjects: Using the Internet as an alternative source of subjects and research environment. $\mathrm{Be}$ havior Research Methods, Instruments, \& Computers, 29, 496-505.

Stanton, J. M. (1998). An empirical assessment of data collection using the Internet. Personnel Psvchology, 51, 709-725.

Thayer, R. E., Newman. J. R., \& McClain, T. M. (1994). Selfregulation of mood: Strategies for changing a bad mood, raising energy, and reducing tension. Journal of Personality \& Social Psychologv, 67, 910-925.

Tseng, H., Tiplady, B., Macleod, H. A., \& Wright, P. (1998). Computer anxiety: A comparison of pen-based personal digital assistants, conventional computer and paper assessment of mood and performance. British Journal of Psvchology, 89, 599-610.

Wilson, F. R., GeNCO, K. T., \& YAGER, G. G. (1986). Assessing the equivalence of paper-and-pencil vs. computerized tests: Demonstration of a promising methodology. Computers in Human Behavior, $\mathbf{1}$, 265-275.

(Manuscript received November 16, 1998 revision accepted for publication April 27, 1999.) 